\title{
Development of A Design System for the Sprout Vertical Cultivation Device
}

\author{
GUO $\mathrm{Yi}^{1, \mathrm{a}^{*}}$, ZHANG Guiqin ${ }^{2, b}$ \\ ${ }^{1}$ Department of Horticulture Beijing Vocational College of Agriculture, Beijing China \\ ${ }^{2}$ Beijing Green Valley Sprouts Limited Liability Company, Beijing China \\ aguoyibvca@163.com, bzhangguiqin0405@sina.com
}

Keywords: sprout vertical cultivation device, Visual Basic; SolidWorks; redevelopment.

\begin{abstract}
A design system for the sprout vertical cultivation device has been developed with Visual Basic and SolidWorks redevelopment technology. This system features a simple user interface and easy operation. Practical applications show that the system can work out the manufacturing materials required and update the 3D models for parts and assemblies according to such design parameters as the length and width of the seedling tray, the number of seeding trays of each layer and the number of layers input by the user. It has practical value in production and has provided reference for development of other systems.
\end{abstract}

\section{Introduction}

Sprouts become more and more popular with consumers in the market owing to their fresh quality, unique taste, rich nutrition and no pollution. They can meet requirements on green foods and thus have grown into fashionable vegetables. In sprouts factories, vertical cultivation devices are adopted. Such devices require substantive design workload and complicated calculation of manufacturing materials, but have no design system available yet. To simplify the design of the sprout vertical cultivation devices, a design system for the sprout vertical cultivation devices has been designed with Visual Basic and SolidWorks redevelopment technology. The system can calculate the materials required and update the 3D models.

SolidWorks is 3D CAD software based on the Win platform. It supports parameterization and characteristic modelling technology and is capable of creating entities with complicated shapes in a convenient and fast manner. It can also realize the parameterized drive for the entities. More importantly, SolidWorks has provided a lot of API functions for redevelopment, so that users can use Visual Basic and C to make redevelopment of SolidWorks [1-7].

GUO Yi and et al. developed the design system of vegetable solar green house [8]; FENG Zhimeng and et al. developed the high speed spindle system [9]; YAO Hui and et al. developed the 3D standard part library of rolling bearings [10]; GUAN Feng and et al. developed the six-bar linkage of shaper[11]; ZHANG Xiaoying and et al. developed the rapid design system of the shipbuilding gantry crane trolleys[12]; WANG Wenbin and et al. developed CAD system of helical elliptical gear [13]; LIU Tairan and et al. developed the tooth-modification system of straight bevel gear [14]; JIANG Zhennan and et al. developed the parametric design of flameproof enclosure [15]; DONG Huajun developed the design system for contact of vacuum circuit breaker [16]; HU Chaobin and et al. developed the parametric design system of hydraulic cylinder [17]; LI Changping and et al. developed the design system of the DTH drilling bits [18]; YI Huijun and et al. developed the system for chemical standard parts [19]; and ZHANG Shuangquan and et al. developed the parametric design system for the fan tower [20].

To sum up, Visual Basic works as a development tool to make redevelopment to the SolidWorks software. It can realize visualized interface operation and automatically create the 3D model in SolidWorks, to increase the design quality and efficiency. Currently, there are many studies on redevelopment, but no study has been found on redevelopment of the sprout vertical cultivation device and the design system for calculating manufacturing materials required. 


\section{Development of the Design System}

Requirements. Based on user demand, the design system shall be able to calculate manufacturing materials required according to the physical dimensions of the sprout vertical cultivation device given by the user and automatically create 3D models for each part and the assembly. Besides, the design interface shall be user-friendly, easy to operate and simple to use.

Development Flow. The design system has been developed through: firstly, using SolidWorks to build 3D models for each part and assembly of the sprout vertical cultivation device; secondly, applying Visual Basic for programming to create the user interface, working out the quantity of manufacturing materials required, updating dimensional variables of the 3D models to generate the 3D models to meet the user's demand. The detailed development flow is shown in Fig. 1.

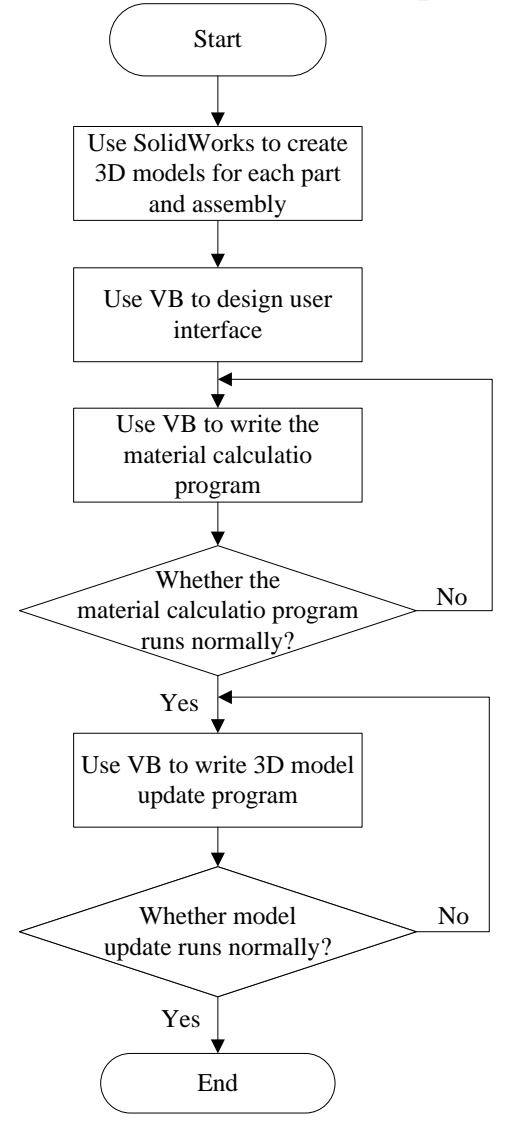

Fig. 1 Development Flow

Create 3D Models for the Device. The sprout vertical cultivation device is composed of the base, side beam, rack and seedling tray. The base is composed of the side bearer, crossbeam and wheels, all being bolted to each other. In manufacturing, the seedling tray, wheels and bolts adopt the universal products. Other materials are shown in Table 1. The device can be either vertical or expanded. The vertical state is applicable to the forcing of sprouting of sprouts and the expanded state to the growth period of the sprout.

Table 1 Materials Required by the Sprout Vertical Cultivation Device

\begin{tabular}{ccc}
\hline Part name & Material name & Material specification \\
\hline Side bearer & Square steel & $30 \mathrm{~mm} \times 30 \mathrm{~mm}$ \\
Crossbeam & Angle steel & $30 \mathrm{~mm} \times 30 \mathrm{~mm}$ \\
Side beam & Square steel & $30 \mathrm{~mm} \times 30 \mathrm{~mm}$ \\
Rack & Angle steel & $30 \mathrm{~mm} \times 30 \mathrm{~mm}$ \\
Rack & Flat steel & $30 \mathrm{~mm} \times 3 \mathrm{~mm}$ \\
\hline
\end{tabular}

According to composition and materials of the device, the SolidWorks software is used to build 3D models for the side bearer, crossbeam, side beam, rack and seedling tray (Fig. 2). Then the 3D model for the sprout vertical cultivation device is built according to the assembly relation (Fig. 3). 
Create the User Interface. The user interface is composed of the design parameter input box, display frame for the calculation results of materials required, calculation button for materials required, the 3D model update button and the exit button. Visual Basic is used to build the window document, add objects (frame, label, textbox, buttons), edit attribute of each widget and then create the user interface, as shown in Fig. 4.

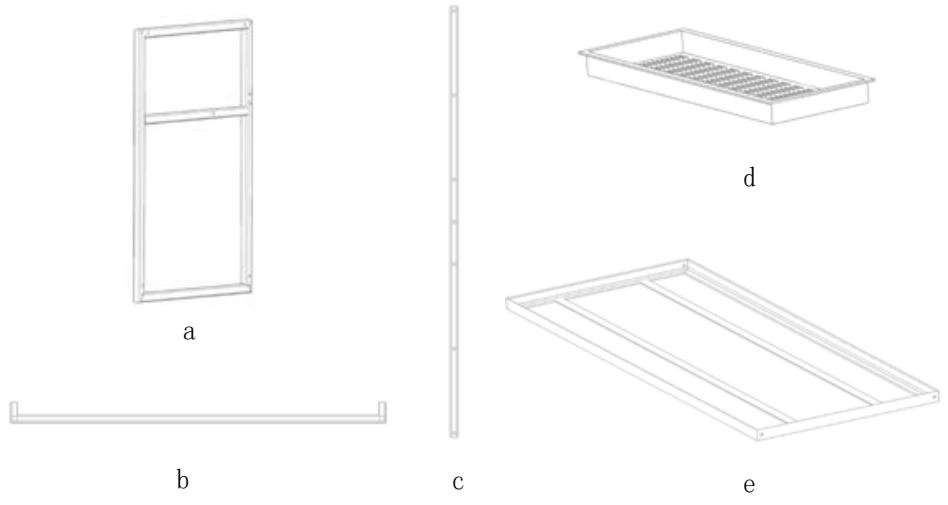

Fig. 2 3D Model of Each Part

a-Side bearer; b-Crossbeam; c-Side beam; d-seedling tray; e-Rack

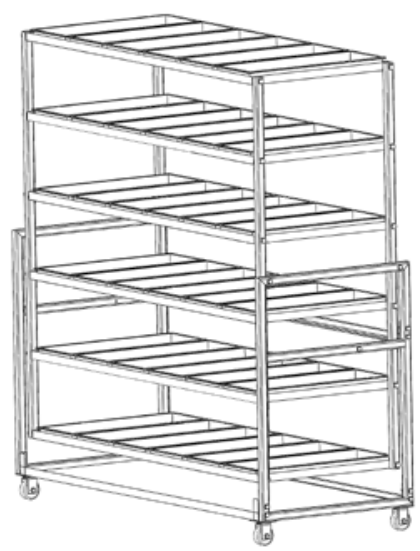

Fig. 3 3D Model of the Sprout Vertical Cultivation Device

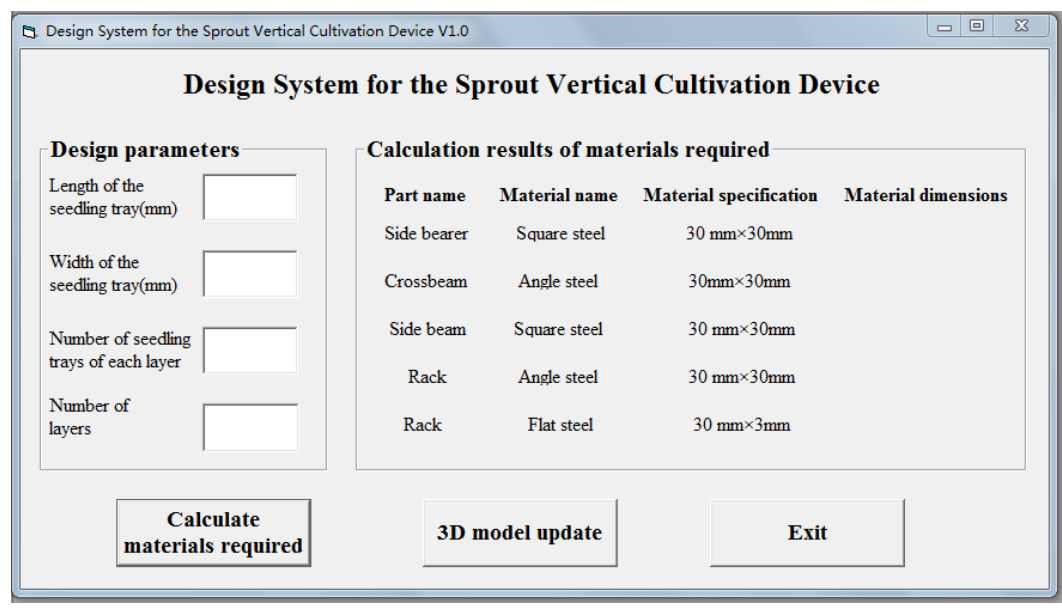

Fig. 4 User Interface

Prepare Material Calculation Program. The manufacturing material calculation program makes the calculation according to functions provided by Visual Basic, arithmetic operators and the expressions, to work out materials required. Namely, Visual Basic is used for programming. The dimension of each part is worked out according to the length and width of the seedling tray, number 
of seedling trays of each layer and number of layers; also the dimensions of materials required by each part will be calculated. Since wheels are universal parts, they are not listed in the results.

Prepare the 3D Model Update Program. The 3D model update program is to update parts and assemblies of the sprout vertical cultivation device according to design parameters input by the user. Namely, Visual Basic is used for programming. The dimension of each part is calculated according the length and width of the seedling tray and number of seedling trays of each layer and number of layers input by the user and API functions of SolidWorks are called to update the 3D models of each part. Then, with the full correlation of SolidWorks documents, after update of the part models, the dimensions are directly transferred to the assembly files to complete update of the assemblies and 3D models.

\section{Applications}

This system has been applied to a sprout vertical cultivation device as follows:

(1) Use Visual Basic to activate the user interface;

(2) Input the length $60 \mathrm{~mm}$ and width $23 \mathrm{~mm}$ of the seedling tray, 6 seedling trays of each layer and 6 layers in the corresponding box on the interface;

(3) Click the " Calculate materials required " button so that the system will automatically calculate the materials required according to parameters input, with results shown in Fig. 5.

(4) Click the "3D model update " button so that the system will automatically update 3D models for each part and assembly according to parameters input and save files. The design results (the expanded state) are shown in Fig. 6.

(5) Click " Exit " button to exit the system.

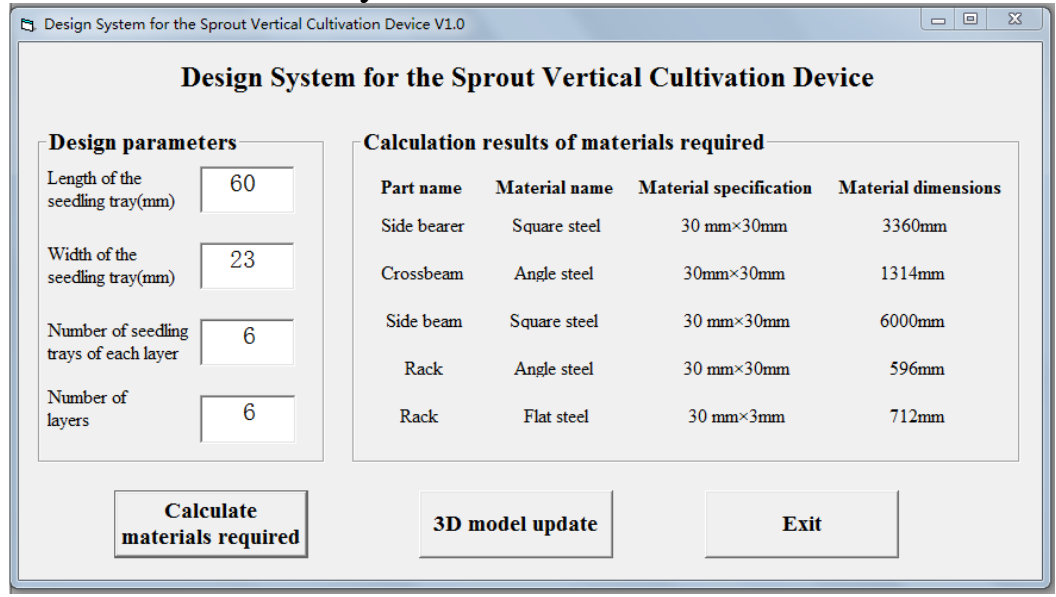

Fig. 5 Results of Materials Required

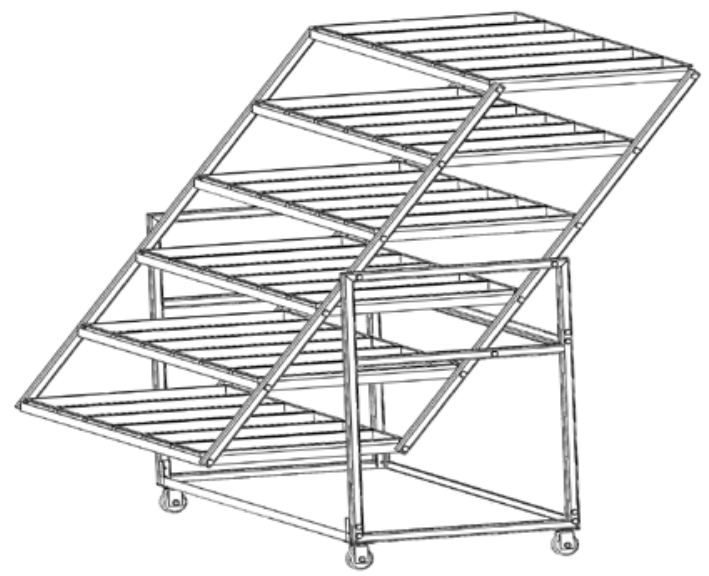

Fig. 6 Design Results of the Sprout Vertical Cultivation Device 


\section{Summary}

The design system for the sprout vertical cultivation device has been developed with Visual Basic and SolidWorks redevelopment technology. Applications have shown that this system features a simple user interface and easy operation. It can work out the manufacturing materials required and update the 3D models for parts and assemblies according to such design parameters as the length and width of the seedling tray, the number of seeding trays of each layer and the number of layers input by the user. It is capable of calculating the materials required and updating 3D models. Thus it has practical value in production and has provided reference for development of other systems.

\section{References}

[1] Hang ZHOU, Xueming QIAN, Lan DUAN, Linhan LI, Development of parametric design system for hardware. Mechanical Engineering \& Automation, 02(2014) 78-79.

[2] Zhilin WU, Kaihuan ZHANG, Secondary development of dimension-driven model construction based on Solidworks. Computer Era, 01(2013) 14-17.

[3] Min SHI, Xinyuan CHEN, Congchang ZHAN, Lafu Lu, Zhiwu HUANG, Lin ZUO, Parametric design of AGC hydraulic cylinder based on SolidWorks. Journal of Wuhan University of Science and Technology, 04(2014) 297-299.

[4] Yahui LIU, Shumei LIU, Qiuhua YU, Parametric Design of Stamping Die Guide Parts Based on SolidWorks, Journal of Shanghai University of Engineering Science, 01(2014) 63-67.

[5] Xianjun JIN, Zanpeng WEN, Weigang SONG, Research on design and simulation of pneumatic rock drill based on secondary development of SolidWorks, Mining \& Processing Equipment, 09(2013) 18-21.

[6] Chao SUO, Yuxiang LI, Shuzhong LIN, Secondary development of SolidWorks parametric design based on Visual Basic language, Manufacturing Automation, 15(2013) 137-140.

[7] Xuan WANG, Xiaobo DAI, Yude DONG, Parametric design of ceramic mould based on SolidWorks. Journal of Xi'an Polytechnic University, 03(2014), 293-297.

[8] Yi GUO, Zhiqiang LI, Research on the Design System of Vegetable Solar Greenhouse, Hubei Agricultural Sciences, 19(2013)4800-4803.

[9] Zhiming FENG, Huijuan SUN, The Development of High Speed Spindle System Based on SolidWorks, Machinery Design \& Manufacture, 02(2013)26-28.

[10] Hui YAO, Yan CAO, Yu BAI, Jiang DU, Development of 3D standard part library of rolling bearing Driven with Standard Configuration, Journal of Xi'An Technological University, 06(2014)465-469.

[11] Feng GUAN, Chuanxi ZHOU, Jiugang YU, Development of the Software for Six-bar Linkage of Shaper Based on Visual Basic, Machine Tool \& Hydraulics, 20(2013)23-25.

[12] Xiaoying ZHANG, Mingjing HE, LI Rong, Rapid design system development of shipbuilding gantry crane trolleys based on SolidWorks, Machinery, 10(2014)60-65.

[13] Wenbin WANG, Yanfei Ren, Yancang JIANG, Study on CAD system of helical elliptical gear, Journal of Mechanical Transmission, 06(2014)78-81.

[14] Tairan LIU, Huanyong CUI, Haiyan WANG, Xijie TIAN, The Tooth-Modification System of Straight Bevel Gear Based on SolidWorks, Journal of University of Jinan (Science and Technology), 06(2014)446-451. 
[15] Zhennan JIANG, Qiaoming GU, Huafeng MAO, Haifeng MO, Yingjie JIANG, Parametric Design of Flameproof Enclosure Based on SolidWorks, Coal Mine Machinery, 03(2014)192-194.

[16] Huajun Dong, Xuemei JIANG, Yingjie GUO, Kan ZANG, Fangzhun GUO, Parametric Design for Contact of Vacuum Circuit Breaker Based on Solid Works, Chinese Journal of Vacuum Science and Technology, 10(2014)1135-1139.

[17] Chaobin HU, Lin XIA, Peng HUANG, Parametric Design of Hydraulic Cylinder Based on Visual Basic and Solidworks, Coal Mine Machinery, 12(2014)254-256.

[18] Changping LI, Jianhua RAO, Junlin HU, Design and realization of DTH drilling bits solidworks secondary development system based on Visual Basic.NET, Manufacturing Automation, 16(2013)96-99.

[19] Huijun YI, Yingfa YANG, Further Development of Chemical Standard Parts Based on SolidWorks, Control and Instruments In Chemical Industry, 05(2013)663-666.

[20] Shuangquan ZHANG, Jingbo LIU, Yize YUN, Hailong WANG, Parametric Design of Wind Turbine Tower Based on Secondary Development of SolidWorks, Machine Building \& Automation, 02(2015)143-145. 